TRANSACTIONS OF THE

AMERICAN MATHEMATICAL SOCIETY

Volume 351, Number 10, Pages 4027-4042

S 0002-9947(99)02278-3

Article electronically published on April 20, 1999

\title{
ON THE DEPTH OF THE TANGENT CONE AND THE GROWTH OF THE HILBERT FUNCTION
}

\author{
JUAN ELIAS
}

\begin{abstract}
For a $d$-dimensional Cohen-Macaulay local ring $(R, \mathbf{m})$ we study the depth of the associated graded ring of $R$ with respect to an $\mathbf{m}$-primary ideal $I$ in terms of the Vallabrega-Valla conditions and the length of $I^{t+1} / J I^{t}$, where $J$ is a $J$ minimal reduction of $I$ and $t \geq 1$. As a corollary we generalize Sally's conjecture on the depth of the associated graded ring with respect to a maximal ideal to $\mathbf{m}$-primary ideals. We also study the growth of the Hilbert function.
\end{abstract}

\section{INTRODUCTION}

Let $(R, \mathbf{m})$ be a $d$-dimensional Cohen-Macaulay local ring with maximal ideal $\mathbf{m}$. Let $I$ be an $\mathbf{m}$-primary ideal of $R$, and $J$ a minimal reduction of $I$. We denote by $G(I)=\bigoplus_{n \geq 0} I^{n} / I^{n+1}$ the associated graded ring to $R$ with respect $I$, and call it the tangent cone for short.

In [Abh67] Abhyankar proved that if $e_{0}=e_{0}(R)$ is the multiplicity of $R$ and $b=\operatorname{dim}_{\mathbf{k}}\left(\mathbf{m} / \mathbf{m}^{2}\right)$ is its embedding dimension, then we have

Abhyankar's bound. $e_{0} \geq b-d+1$.

Sally in a long series of papers studied the depth of the tangent cone in terms of the difference $\varepsilon=e_{0}-(b-d+1) \geq 0$; see [Sal77], [Sal79a], [Sal79c], [Sal80a], [Sal80b], and [Sal83]. She proved that if $\varepsilon=0$ then $G(\mathbf{m})$ is Cohen-Macaulay, [Sal77], and proposed the following conjecture, [Sal83]:

Sally's Conjecture. If $\varepsilon=1$, then $\operatorname{depth}(G(\mathbf{m})) \geq d-1$.

This conjecture was proved by Rossi and Valla, [RosV96a], and independently by Wang, [Wan97]. Valla in [Val79] extended Abhyankar's bound to m-primary ideals $I$, and proved that the tangent cone $G(I)$ is Cohen-Macaulay if the bound is reached. Using the device of Sally's module and Ratllif-Rush closure, several results on the depth of the tangent cone of $\mathbf{m}$-primary ideals appeared in [Sal92], [Vas94], and [Gue95], [Gue94], [Vaz95]. It is worthwhile to recall that Huckaba, [Huc97], following [RosV96a], proved a generalization of Sally's conjecture for $\mathbf{m}$-primary ideals.

Sally studied the case $\varepsilon=0,1$ under stronger conditions, proving that if $R$ is a Gorenstein ring then $G(\mathbf{m})$ is also Gorenstein, [Sal80a], [Sal80b].

The aim of this paper is to generalize and to present an unified approach to some results on the depth of the tangent cone and the growth of the Hilbert function

Received by the editors June 24, 1997.

1991 Mathematics Subject Classification. Primary 13A30, 13D40, 13 H10.

Partially supported by DGICYT PB94-0850. 
appearing in [Sal77], [Val79], both on Abhyankar's inequality, and in [RosV96a], [Wan97], and [Huc97], on Sally's conjecture. We also cover some more results appearing in [Sal92], [Vas94], and [Gue95], [Gue94], [Vaz95]. See Corollary 2.8 for more details.

Let us recall that Vallabrega and Valla discovered the conditions under which $G(I)$ is Cohen-Macaulay. They proved that, given a minimal reduction $J$ of $I$, then $G(I)$ is Cohen-Macaulay if and only if for all $n \geq 0$ the $n$-th Vallabrega-Valla condition holds, [VV78]:

Vallabrega-Valla condition $\mathcal{V}_{n}^{2} . \quad I^{n} \cap J=I^{n-1} J$.

The key idea of this paper is to consider ideals $I$ for which the Vallabrega-Valla conditions $\mathcal{V}_{n}^{2}$ hold up to some integer $t \geq 1$. The main result of this paper is:

Theorem 2.7. Let $R$ be a d-dimensional, $d \geq 1$, Cohen-Macaulay local ring. Let $I$ be an $\mathbf{m}$-primary ideal of $R$, and $J=\left(x_{1}, \ldots, x_{d}\right)$ a minimal reduction of $I$. Let us assume that there exists an integer $t \geq 1$ such that

- $I, J$ satisfy the condition $\mathcal{V}_{n}^{2}$ for $n=0, \ldots, t$, and

- length $\left(I^{t+1} / J I^{t}\right)=\delta \leq \operatorname{Min}\{d-1,1\}$.

Then

$$
d \geq \operatorname{depth}(G(I)) \geq d-\delta .
$$

If $t \geq e_{0}(I)-1$, then $G(I)$ is Cohen-Macaulay.

We prove this result by generalizing and extending results of [Sal83], [RosV96a], and [Huc97] mainly, to $\mathbf{m}$-primary ideals and considering higher Vallabrega-Valla conditions. Notice that the main result of [RosV96a] is the particular case $I=\mathbf{m}$, $t=1$, and [Huc97] is $t=2$ and $I$ an $\mathbf{m}$-primary ideal.

We also prove that the conditions of the above result can be fulfilled, Proposition 2.9. This means that for all $d, t \geq 1$, and $\delta \leq 1$ there exists a $d$-dimensional Cohen-Macaulay local ring such that its maximal ideal $\mathbf{m}$ admits a $d$-generated minimal reduction $J$ satisfying both conditions of the main result and $\operatorname{depth}(G(\mathbf{m}))$ $=d-\delta$.

In $\S 3$ we compute the Hilbert function of ideals under the conditions above, Proposition 3.3. Moreover, if length $\left(I^{t+1} / J I^{t}\right) \leq 2$ then the Hilbert function of $I$ has a non-decreasing $(d-1)$-derivative, Proposition 3.2. We end the paper by applying these results to the one-dimensional case.

Notations. Without loss of generality we may assume that the residue field $\mathbf{k}=$ $R / \mathbf{m}$ is infinite. We will denote by $H_{I}$ the Hilbert function of $I$,

$$
H_{I}(n)=\text { length }_{R}\left(I^{n} / I^{n+1}\right),
$$

$n \geq 0$. It is well known that there exists a polynomial (the Hilbert polynomial) $h_{I} \in \mathbb{Q}[Z]$ such that $H_{I}(n)=h_{I}(n)$ for $n \gg 0$, and that can be written in the following form

$$
h_{I}(Z)=\sum_{i=0}^{d-1}(-1)^{i} e_{i}(I)\left(\begin{array}{c}
Z+d-i-1 \\
d-i-1
\end{array}\right) .
$$

Let $\mathcal{F}=\left\{\mathcal{F}_{n}\right\}_{n \geq 0}$ be a Hilbert filtration of $R$, i.e. there exist an $\mathbf{m}$-primary ideal $K$ and an integer $n_{0}$ such that $\mathcal{F}_{n+1}=K \mathcal{F}_{n}$ for all $n \geq n_{0}$. We will denote by $r(\mathcal{F}, K)$ the reduction index of $\mathcal{F}$ with respect to $K$ : the least integer $r$ such $\mathcal{F}_{n+1}=K \mathcal{F}_{n}$ for all $n \geq r$. 
We will denote by $\tilde{I}$ the Ratliff-Rush closure of $I$; see [HLS92] for the main properties of Ratliff-Rush closure. $\mathcal{R}(\mathcal{F})=\bigoplus_{n \geq 0} \mathcal{F}_{n} T^{n}$ will be the Rees algebra associated to a filtration $\mathcal{F}=\left\{\mathcal{F}_{n}\right\}_{n \geq 0}$. If $\mathcal{F}=\left\{I^{n}\right\}_{n \geq 0}$ (resp. $\mathcal{F}=\left\{\widetilde{I^{n}}\right\}_{n \geq 0}$ ) then we write $\mathcal{R}(\mathcal{F})=\mathcal{R}(I)($ resp. $\mathcal{R}(\mathcal{F})=\widetilde{\mathcal{R}}(I))$. We write $r=r(I, J)=r\left(\left\{I^{n}\right\}_{n \geq 0}, J\right)$ for the reduction number of $I$ with respect to $J$.

\section{The Depth of the tangent Cone}

Since our purpose is to prove that the tangent cone has at least depth $d-1$, and the conditions of Theorem 2.7 are preserved modulo a superficial sequence of $I$, most of the time we can proceed, using "Sally's machine", by considering a two dimensional local ring $R$. See [Sal79b], [HM94], Lemma 2.2 for a proof, and see also [Ito95], Lemma 1(2).

From now on we will denote by $\mathcal{D}(I)$ the $\mathcal{R}(I)$-graded module $\widetilde{\mathcal{R}}(I) / \mathcal{R}(I)$. Notice that if we denote by $\mathcal{R}^{*}=\mathcal{R}^{*}(I)$ the extended Rees algebra associated to $I$, i.e. $\mathcal{R}_{n}^{*}=R$ for $n<0$, and $\mathcal{R}_{n}^{*}=I^{n}$ for $n \geq 0$, then we have, [Bla95],

$$
\mathcal{D}(I) \cong H_{\mathcal{R}^{+}}^{1}\left(\mathcal{R}^{*}\right) \text {. }
$$

Let $J$ be a minimal reduction of $I$. We will denote by $\nu_{n}=\nu_{n}(I, J)$ the minimal number of generators of the $R$-module

$$
\left(\frac{\mathcal{D}(I)}{J T \mathcal{D}(I)}\right)_{n} \cong \frac{\widetilde{I^{n}}}{J I^{n-1}+I^{n}}
$$

$n \geq 0$. We set $\nu=\nu(I, J)=\sum_{n \geq 1} \nu_{n}$.

We denote by $\tilde{r}(I, J)$ the least integer such that $\widetilde{I^{n+1}}=J \widetilde{I^{n}}$; notice that we have $\tilde{r}(I, J) \leq r\left(\left\{\widetilde{I^{n}}\right\}_{n \geq 0}, J\right)$. We will denote by $s(I, J)$ the least integer such that $I^{n+1} \subset J \widetilde{I^{n}}$. It is easy to see that $s(I, J) \leq \tilde{r}(I, J)$. If $x$ is a superficial element of $I$ belonging to $J$, we will denote by $\bar{r}=\bar{r}(I, J)$ the integer $r(I /(x), J /(x))$,

Proposition 2.1. Let $R$ be a d-dimensional Cohen-Macaulay local ring, I an $\mathbf{m}-$ primary ideal, and $J$ a minimal reduction of $I$. Assume that there exists an integer $t \geq 1$ such that the pair $I, J$ satisfies the condition $\mathcal{V}_{n}^{2}$ for $n=0, \ldots, t$. Then, for all $n=0, \ldots, t$,

$$
\nu_{n} \leq \text { length }\left(\widetilde{I^{n}} / J I^{n-1}\right)-\text { length }\left(I^{n} / J I^{n-1}\right) .
$$

Proof. Let us consider the following inequalities:

$$
\begin{aligned}
\nu_{n} & =\nu\left(\frac{\widetilde{I^{n}}}{J \widetilde{I^{n-1}}+I^{n}}\right) \\
& \leq \text { length }\left(\frac{\widetilde{I^{n}}}{J \widetilde{I^{n-1}}+I^{n}}\right)=\text { length }\left(\widetilde{I^{n}}\right)-\operatorname{length}\left(\frac{J \widetilde{I^{n-1}}+I^{n}}{I^{n}}\right) .
\end{aligned}
$$

Since the pair $I, J$ satisfies $\mathcal{V}_{n}^{2}$ for $n=0, \ldots, t$, we get

$$
\widetilde{J I^{n-1}} \cap I^{n} \subset J \cap I^{n}=J I^{n-1},
$$

so we have $J \widetilde{I^{n-1}} \cap I^{n}=J I^{n-1}$ and

$$
\begin{aligned}
\nu_{n} & \leq \text { length }\left(\frac{\widetilde{I^{n}}}{I^{n}}\right)-\text { length }\left(\frac{J \widetilde{I^{n-1}}}{J I^{n-1}}\right) \\
& =\text { length }\left(\frac{\widetilde{I^{n}}}{J I^{n-1}}\right)-\text { length }\left(\frac{I^{n}}{J I^{n-1}}\right) .
\end{aligned}
$$


Let us recall the following result of Huckaba, where $e_{1}(I)$ is computed for $d=1,2$, [HM94], Corollary 4.13, and [Huc96], Theorem 3.1.

Proposition 2.2. Let $R$ be a $d$-dimensional Cohen-Macaulay local ring, $I$ an $\mathbf{m}-$ primary ideal of $R$, and $J$ a d-generated reduction of $I$. Then the following conditions hold:

(1) If $d=1$ then $e_{1}(I)=\sum_{n \geq 0}$ length $\left(\frac{I^{n+1}}{J I^{n}}\right)$.

(2) If $d=2$ then $e_{1}(I)=\sum_{n \geq 0}$ length $\left(\frac{\widetilde{I^{n+1}}}{J \widetilde{I}^{n}}\right)$, and the following conditions are equivalent:

(2.1) $\operatorname{depth}(G(I)) \geq 1$,

(2.2) $I^{n}=\widetilde{I^{n}}$ for all $n \geq 1$,

(2.3) $e_{1}(I)=\sum_{n=0}^{r-1}$ length $\left(\frac{I^{n+1}}{J I^{n}}\right)$, with $\quad r=r(I, J)$.

The next result is a generalization to a $\mathbf{m}$-primary ideal and extension to any power of [Huc97], Lemma 2.1, and [RosV96a], Lemma 1.1. We will use this result in the proofs of Theorem 2.7 and Proposition 3.2.

Proposition 2.3. Let $R$ be a d-dimensional, $d \geq 1$, local ring. Let $I$ be an $\mathbf{m}$-primary ideal of $R$, and let $K$ be an ideal of $R$ such that $K \subset I^{m}$ and

$$
\text { length }\left(\frac{I^{m}}{K}\right) \leq 1 \text {. }
$$

Then either $I^{m+1}=K I$, or there exists $g \in I$ such that for all $n \geq m$

$$
I^{n}=I^{n-m} K+\left(g^{n}\right) .
$$

Proof. Let $\lambda=\operatorname{length}_{R}\left(I^{m} / K\right)$. If $\lambda=0$, then we get the result. Assume $\lambda=1$, and let $a_{1}, \ldots, a_{r}$ be a system of generators of $I$. Notice that $r \geq d \geq 1$.

If $r=d=1$, then $I$ is principal and the result becomes clear. Let us assume $r \geq 2$. Hence there exists a degree $m$ multi-index $\beta=\left(\beta_{1}, \ldots, \beta_{r}\right) \in \mathbb{N}^{r}$ such that $a^{\beta}=a_{1}^{\beta_{1}} \ldots a_{r}^{\beta_{r}}$ and

$$
I^{m}=K+\left(a^{\beta}\right) .
$$

After a change of indices we may assume that $\beta_{1}>0$. Since $\lambda=1$ we get that $a^{\beta} \notin K$, and then we can consider the multi-index $\gamma$ of degree $m$ such that $\gamma_{1}$ is the biggest integer such that $a^{\gamma} \notin K$. In particular we have $I^{m}=K+\left(a^{\gamma}\right)$. If $\gamma=(m, 0, \ldots, 0)$, then we get the claim with $g=a$. If $\gamma_{1}<m$, then we can assume that $\gamma_{2}>0$, so $a^{\gamma+(1,0, \ldots, 0)} \in a_{2} K \subset I K$ and

$$
\begin{aligned}
I^{m+1} & =I K+a^{\gamma} I \subset I K+a_{1} I^{m} \\
& \subset I K+a_{1}\left(K+a^{\gamma}\right) \\
& =I K+a_{1} K+\left(a^{\gamma+(1,0, \ldots, 0)}\right) \\
& \subset I K \subset I^{m+1},
\end{aligned}
$$

and then $I^{m+1}=I K$. If $I^{m}=K+\left(g^{m}\right)$, by induction on $n$ it is easy to prove that $I^{n}=I^{n-m} K+\left(g^{n}\right)$ for all $n \geq m$. 
The next result is needed to compute $e_{1}(I)$ under the hypothesis of the main result of this paper and to establish some conditions to assure $\operatorname{depth}(G(I)) \geq 1$. This result is an improvement and generalization of [Sal83], Corollary 5.9, [RosV96a], Corollary 2.3, and [Huc97], Lemma 2.2.

Proposition 2.4. Assume that $(R, \mathbf{m})$ is a 2-dimensional Cohen-Macaulay local ring, $I$ an $\mathbf{m}$-primary ideal, and $J=(x, y)$ is a minimal reduction of $I$. Let $r=r(I, J), s=s(I, J)$, and $\bar{r}=r(I /(x), J /(x))$. Assume that there exists an integer $t \geq 1$ such that

- I, $J$ satisfy the condition $\mathcal{V}_{n}^{2}$ for $n=0, \ldots, t$, and

- length $\left(I^{t+1} / J I^{t}\right) \leq 1$.

Then the following conditions hold:

(1) $e_{1}(I)=\sum_{n=0}^{\bar{r}-1}$ length $\left(I^{n+1} / J I^{n}\right)$.

(2) $\bar{r} \leq r$, and $\bar{r}=r$ if and only if $\operatorname{depth}(G(I)) \geq 1$.

(3) If $\bar{r} \leq t$, then $\operatorname{depth}(G(I))=2$.

(4) If $t<\bar{r} \leq r$, then

$$
t \leq s \leq \tilde{r} \leq \bar{r} \leq r .
$$

(5) If $\operatorname{depth}(G(I)) \geq 1$, then $s=\tilde{r}=\bar{r}=r$.

Proof. (1) Since $x$ is a superficial element for $I$, we have $e_{1}(I)=e_{1}(I /(x))$. From Proposition $2.2(1)$ we obtain

$$
\begin{aligned}
e_{1}(I) & =\sum_{n=0}^{\bar{r}-1} \text { length }\left(\frac{(I /(x))^{n+1}}{(J /(x))(I /(x))^{n}}\right) \\
& =\sum_{n=0}^{\bar{r}-1} \text { length }\left(\frac{I^{n+1}}{J I^{n}+x\left(I^{n+1}: x\right)}\right) .
\end{aligned}
$$

Since the pair $I, J$ satisfies $\mathcal{V}_{n}^{2}$ for $n=1, \ldots, t$, we deduce that

$$
\left(I^{n+1}: x\right)=\left(I^{n+1}: y\right)=I^{n}
$$

for $n=1, \ldots, t-1$. If $\bar{r} \leq t$, then we get

$$
e_{1}(I)=\sum_{n=0}^{\bar{r}-1} \text { length }\left(\frac{I^{n+1}}{J I^{n}}\right) .
$$

If $\bar{r}>t$ and $\bar{r}-1 \geq n \geq t$, then from Proposition 2.3 we get

$$
\begin{aligned}
0 & <\text { length }\left(\frac{(I /(x))^{n+1}}{(J /(x))(I /(x))^{n}}\right) \\
& =\text { length }\left(\frac{I^{n+1}}{J I^{n}+x\left(I^{n+1}: x\right)}\right) \\
& \leq \text { length }\left(\frac{I^{n+1}}{J I^{n}}\right) \leq 1 .
\end{aligned}
$$

Hence $x\left(I^{n+1}: x\right) \subset J I^{n}$ for $n=t, \ldots, \bar{r}-1$. Since $\left(I^{n+1}: x\right)=I^{n}$ for $n=$ $1, \ldots, t-1$, from (F1) and Proposition 2.2 we get (1). 
(2) From the definitions we get $\bar{r} \leq r$. From (1) and Proposition 2.2 (2) we get that $\bar{r}=r$ if and only if $\operatorname{depth}(G(I)) \geq 1$.

(3) Let us assume that $\bar{r} \leq t$. From (1) and Proposition 2.2 (2) we get

$$
\begin{aligned}
0 & =\sum_{n \geq 0} \text { length }\left(\widetilde{\frac{I^{n+1}}{J I^{n}}}\right)-\sum_{n=0}^{\bar{r}-1} \text { length }\left(\frac{I^{n+1}}{J I^{n}}\right) \\
& =\sum_{n=0}^{r-1}\left(\text { length }\left(\widetilde{\frac{I^{n+1}}{J I^{n}}}\right)-\text { length }\left(\frac{I^{n+1}}{J I^{n}}\right)\right)+\sum_{n \geq \bar{r}} \text { length }\left(\widetilde{\frac{I^{n+1}}{J I^{n}}}\right) .
\end{aligned}
$$

From Proposition 2.1 we deduce length $\left(I^{n+1} / J I^{n}\right)=\operatorname{length}\left(\widetilde{I^{n+1}} / J \widetilde{I^{n}}\right)$ for $n=$ $0, \ldots, \bar{r}-1$, and $\widetilde{I^{n+1}}=J \widetilde{I^{n}}$ for $n \geq \bar{r}$. From these equalities and by induction on $n$ we obtain $I^{n}=\widetilde{I^{n}}$ for all $n \geq 1$. Hence $\operatorname{depth}(G(I)) \geq 1$, by Proposition 2.2 (2). From (2) we get that $\bar{r}=r<t$, so the pair $I, J$ satisfies $\mathcal{V}_{n}^{2}$ for all $n \geq 0$. From the Vallabrega-Valla result we get that $\operatorname{depth}(G(I))=2$.

(4) Let us assume $t<\bar{r} \leq r$. If $s<t$ then we have

$$
I^{t} \subset J \widetilde{I^{t-1}} \subset J \text {. }
$$

Since the pair $I, J$ satisfies the condition $\mathcal{V}_{t}^{2}$, we get

$$
I^{t}=I^{t} \cap J=J I^{t-1},
$$

and then $r \leq t-1$, which is a contradiction with the assumption $t<r$. Hence we get $t \leq s$. By definition, $s \leq \tilde{r}$; therefore $t \leq \tilde{r}$.

Let us consider the following inequalities:

$$
\begin{aligned}
0 & \geq-\sum_{n=1}^{t} \nu_{n} \\
& \geq \sum_{n=0}^{t-1}\left(\text { length }\left(\frac{I^{n+1}}{J I^{n}}\right)-\text { length }\left(\widetilde{\frac{I^{n+1}}{J I^{n}}}\right)\right)
\end{aligned}
$$

by Proposition 2.1

$$
=-\sum_{n=t}^{\bar{r}-1} \text { length }\left(\frac{I^{n+1}}{J I^{n}}\right)+\sum_{n=t}^{\tilde{r}-1} \text { length }\left(\widetilde{\frac{I^{n+1}}{J I^{n}}}\right)+\sum_{n \geq \tilde{r}} \text { length }\left(\widetilde{\frac{I^{n+1}}{J I^{n}}}\right)
$$

by (1) and Proposition $2.2(2)$

$$
\geq-(\bar{r}-t)+\sum_{n=t}^{\tilde{r}-1} \text { length }\left(\widetilde{\frac{I^{n+1}}{J I^{n}}}\right)
$$

by Proposition 2.3. 
From this we get

$$
\bar{r}-t \geq \sum_{n=t}^{\tilde{r}-1} \text { length }\left(\widetilde{\frac{I^{n+1}}{J I^{n}}}\right)
$$

so $\bar{r}-t \geq \tilde{r}-t$.

(5) If $\operatorname{depth}(G(I)) \geq 1$, then from (2) we get $r=\bar{r}$. On the other hand $I^{n}=\widetilde{I^{n}}$ for all $n \geq 0$, and so by the definition of $s$ and $\tilde{r}$ we get $s=\tilde{r}=\bar{r}=r$.

Our next result is a generalization of [RosV96a], Proposition 2.4, and [Huc97], Proposition 2.3, to modules.

Proposition 2.5. Let $R$ be a Noetherian ring, and let $K \subset I$ be ideals of $R$. Let $M$ be a graded $\mathcal{R}(I)$-module, of finite length as $R$-module. Let $\mu$ be the minimal number of generators of $M / \mathcal{R}(K)_{+} M$ as $R$-module. Then for all $z \in I$ there exist $e_{i} \in K^{i}, i=1, \ldots, \mu$, such that

$$
z^{\mu}-z^{\mu-1} e_{1}+\cdots+(-1)^{\mu} e_{\mu} \in \operatorname{Ann}_{R}(M) .
$$

Proof. Let $p$ be the biggest integer such that $M_{p} \neq 0$. For all $i=0, \ldots, p$ we pick elements $m_{i, 1}, \ldots, m_{i, \mu_{i}} \in M_{i}$ such that their cosets in

$$
\left(\frac{M}{\mathcal{R}(K)_{+} M}\right)_{i} \cong \frac{M_{i}}{K^{i} M_{0}+K^{i-1} M_{1}+\cdots+K M_{i-1}}
$$

form a minimal system of generators as $R$-module. We have $\mu=\sum_{i=0}^{p} \mu_{i}$.

If $z$ is an element of $I$, then there exist elements $c_{i, n}^{j, k} \in K^{i-j+1}$ such that

$$
z m_{i, n}=\sum_{j=0}^{p} \sum_{k=1}^{\mu_{j}} c_{i, n}^{j, k} m_{j, k}
$$

with $c_{i, n}^{j, k}=0$ if $j>i+1$. Let $\Lambda$ be the set of pairs $\alpha=(i, n)$ such that $i=0, \ldots, p$, $n=1, \ldots, \mu_{i}$. We consider $\Lambda$ endowed with the lex-ordering. We set $\alpha^{1}=i$, and $\alpha^{2}=n$.

If we denote by $C$ the $\mu \times \mu$ matrix $C=\left(c_{\beta}^{\alpha}\right)_{\alpha, \beta \in \Lambda}$, then we can write

$$
\left(z I d_{\mu \times \mu}-C\right) A=0,
$$

where $A$ is the $\mu \times 1$ matrix $A=\left(m_{\alpha}\right)_{\alpha \in \Lambda}$. Hence we have

$$
\operatorname{det}\left(z I d_{\mu \times \mu}-C\right) \in A n n_{R}(M) .
$$

On the other hand,

$$
\operatorname{det}\left(z I d_{\mu \times \mu}-C\right)=z^{\mu}-z^{\mu-1} e_{1}+\cdots+(-1)^{\mu} e_{\mu},
$$

where $e_{r}$ is the sum of all $r \times r$ minors of $C$ correponding to sub- $r \times r$-matrices of $C$ symmetric with respect to the diagonal of $C$. Notice that $e_{1}$ is the trace of $C$ and $e_{\mu}$ is the determinant of $C$. Let us consider $E=\operatorname{det}\left(\left(c_{\alpha_{j}}^{\alpha_{i}}\right)_{i, j=1, \ldots, r}\right)$, an $r \times r$ minor of $C$. Since

$$
c_{\alpha_{\sigma(1)}}^{\alpha_{1}} \ldots c_{\alpha_{\sigma(r)}}^{\alpha_{r}} \in K^{r}
$$

where $\sigma$ is a permutation of $\{1, \ldots, r\}$, we get $E \in K^{r}$. Hence $e_{i} \in K^{i}, i=0, \ldots, \mu$, and we are done.

In the next result we will apply the last proposition to $M=\mathcal{D}(I)$. 
Proposition 2.6. Let $R$ be a d-dimensional, $d \geq 1$, Cohen-Macaulay local ring. Let $I$ be an $\mathbf{m}$-primary ideal of $R$, and $J$ a minimal reduction of $I$. Let us assume that there exists an integer $t \geq 1$ such that length $\left(I^{t+1} / J I^{t}\right) \leq 1$. If $t \leq s$ then

$$
r \leq \nu+s
$$

Proof. From Proposition 2.3 there exists $g \in I$ such that for all $n \geq t$

$$
I^{n+1}=J I^{n}+\left(g^{n+1}\right) .
$$

Notice that $r \leq \nu+s$ if and only if $g^{\nu+s+1} \in J I^{\nu+s}$. From the definition of $s$ we have

$$
g^{s+1} \in I^{s+1} \subset J \widetilde{I^{s}} .
$$

If we apply the last result to $K=J, M=\mathcal{D}(I), \mu=\nu$, and $z=g$, we get that there exists $h \in J I^{\nu-1}$ such that

$$
\left(g^{\nu}-h\right) \widetilde{I^{n}} \subset I^{\nu+n}
$$

for all $n \geq 0$. Hence we have

$$
g^{s+1+\nu}-g^{s+1} h=g^{s+1}\left(g^{\nu}-h\right) \in J \widetilde{I^{s}}\left(g^{\nu}-h\right) \subset J I^{\nu+s},
$$

so we get $g^{\nu+s+1} \in J I^{\nu+s}$.

Theorem 2.7. Let $R$ be a $d$-dimensional, $d \geq 1$, Cohen-Macaulay local ring. Let $I$ be an $\mathbf{m}$-primary ideal of $R$, and $J=\left(x_{1}, \ldots, x_{d}\right)$ a minimal reduction of $I$. Let us assume that there exists an integer $t \geq 1$ such that

- $I, J$ satisfy the condition $\mathcal{V}_{n}^{2}$ for $n=0, \ldots, t$, and

- length $\left(I^{t+1} / J I^{t}\right)=\delta \leq \operatorname{Min}\{d-1,1\}$.

Then

$$
d \geq \operatorname{depth}(G(I)) \geq d-\delta .
$$

If $t \geq e_{0}(I)-1$, then $G(I)$ is Cohen-Macaulay.

Proof. If $\delta=$ length $\left(I^{t+1} / J I^{t}\right)=0$, the pair $I, J$ satisfies $\mathcal{V}_{n}^{2}$ for all $n \geq 0$. From the Vallabrega-Valla result we get $\operatorname{depth}(G(I))=d$. In particular we may assume $d \geq 2$ and length $\left(I^{t+1} / J I^{t}\right)=1$. We need to prove that

$$
\operatorname{depth}(G(I)) \geq d-1 \text {. }
$$

An easy computation shows that the conditions of the claim are preserved modulo superficial elements. Hence we may assume that $d=2$ (see the beginnig of this section).

From Proposition $2.4(2),(3),(4)$, and Proposition 2.6 we may assume that

$$
t \leq s \leq \tilde{r} \leq \bar{r} \leq r \leq \nu+s
$$

By Proposition 2.4 (2) we only need to prove that $\nu+s \leq \bar{r}$. Let us consider the following inequalities:

$$
\begin{aligned}
\nu & =\sum_{n \geq 1} \nu_{n} \\
& =\sum_{n=1}^{t} \nu_{n}+\sum_{n=t+1}^{s} \nu_{n}+\sum_{n \geq s+1} \nu_{n}
\end{aligned}
$$


$\leq \sum_{n=0}^{t-1}\left(\right.$ length $\left(\widetilde{\frac{I^{n+1}}{J I^{n}}}\right)-$ length $\left.\left(\frac{I^{n+1}}{J I^{n}}\right)\right)+\sum_{n=t+1}^{s} \nu_{n}+\sum_{n \geq s}$ length $\left(\widetilde{\frac{I^{n+1}}{J I^{n}}}\right)$

by Proposition 2.1 and the definition of $s$

$$
=\sum_{n=t}^{\bar{r}-1} \text { length }\left(\frac{I^{n+1}}{J I^{n}}\right)-\sum_{n=t}^{s-1} \text { length }\left(\frac{\widetilde{I^{n+1}}}{J \widetilde{I^{n}}}\right)+\sum_{n=t+1}^{s} \nu_{n}
$$

by Proposition 2.2 and Proposition 2.4 (1)

$$
\begin{aligned}
& \leq(\bar{r}-t)-\sum_{n=t}^{s-1} \text { length }\left(\widetilde{\frac{I^{n+1}}{J I^{n}}}\right)+\sum_{n=t+1}^{s} \nu_{n} \\
& =(\bar{r}-t)+\sum_{n=t+1}^{s}\left(\nu_{n}-\text { length }\left(\frac{\widetilde{I^{n}}}{J I^{n-1}}\right)\right)
\end{aligned}
$$

and by the definition of $s$ we get

$$
\leq(\bar{r}-t)+(t-s)=\bar{r}-s .
$$

Hence we have $\nu+s \leq \bar{r}$, and so $\operatorname{depth}(G(I)) \geq 1$.

Let us assume $t \geq e_{0}(I)-1$, and $d \geq 2$. Since the conditions of the claim are preserved modulo superficial elements, we may assume $d=2$. Let us consider $\bar{R}=R /\left(x_{2}\right), \bar{I}=I /\left(x_{2}\right)$, and $\bar{J}=J /\left(x_{2}\right)$. Since $\bar{R}$ is a one-dimensional CohenMacaulay local ring, we have $e_{0}(I)-1=e_{0}(\bar{I})-1 \geq \bar{r}$, by [SV74], Theorem 2.5, and [ES76], Corollary 3 to Theorem 2. From Proposition 2.4, (3), we get the claim.

Next, we will collect some results that can be obtained as corollaries of Theorem 2.7 .

Corollary 2.8. Under the hypothesis of Theorem 2.7, we denote by $\delta$ the integer $\delta=\operatorname{length}_{R}\left(I^{t+1} / J I^{t}\right)$, and $\rho=\operatorname{depth}(G(I))$. Then the following results hold:

(1) [Sal77], Theorem 2:

$I=\mathbf{m}, t=1, \delta=0$ implies $\rho=d$,

(2) [Val79], Theorem 1:

$t=1, \delta=0$ implies $\rho=d$,

(3) [Vas94], Proposition 2.6(a), and [Gue95], Corollary 2.3(a):

$I^{3}=J I^{2}, t=1, \delta=1$ implies $\rho \geq d-1$,

(4) Sally's conjecture, [Sal83], proved by Rossi and Valla, [RosV96a], and Wang [Wan97]:

$I=\mathbf{m}, t=1, \delta=1$ implies $\rho \geq d-1$,

(5) [Huc97], Theorem 2.4:

$t=2$ implies $\rho \geq d-1$. 
Proof. For the first three results we only need to recall that

$$
\text { length }\left(I^{2} / J I\right)=e_{0}(I)-\text { length }\left(I / I^{2}\right)+(d-1) \text { length }(R / I)
$$

[Val79], Lemma 1.

Notice that Corollary 2.8 (3) was proved also in [Sal92], Lemma 2.3, [Gue94], Corollary 3.3, and [Vaz95], Corollary 3.1.4, with the assmption $r(I, J)=2$.

In the next result we will prove that the conditions of Theorem 2.7 can be fulfilled using the techniques developed in [Eli93a]. Let $S=\mathbf{k}[x, y, z]_{(x, y, z)}$, and let $\left(\mathbf{k}^{3}, 0\right)=\operatorname{Spec}(S)$ be the $\mathbf{k}$-scheme defined by $S$. A curve singularity $X$ is a one-dimensional closed Cohen-Macaulay sub-scheme of $\left(\mathbf{k}^{3}, 0\right)$, i.e. $X=\mathbf{S p e c}(S / I)$ where $I=I(X)$ is a height two perfect ideal of $S$.

Proposition 2.9. For all $d \geq 1, t \geq 1, \delta \in\{0,1\}$, and $\varepsilon \in\{d-\delta, d\}$ there exists a d-dimensional Cohen-Macaulay local ring $(R, \mathbf{m})$ such that $\mathbf{m}$ has a $d$-generated minimal reduction $J$, and

- $\mathbf{m}, J$ satisfy the condition $\mathcal{V}_{n}^{2}$ for $n=0, \ldots, t$,

- length $\left(\mathbf{m}^{t+1} / J \mathbf{m}^{t}\right)=\delta \leq 1$, and

- $\operatorname{depth}(G(\mathbf{m}))=\varepsilon$.

Proof. Case $\delta=0$. From [Eli93a], Proposition 2.2, we deduce that the union of $\left(\begin{array}{c}t+2 \\ 2\end{array}\right)$ generic straight lines through the origin of $\left(\mathbf{k}^{3}, 0\right)$ is a curve singularity $X$ with maximal Hilbert function, i.e.

$$
H_{X}(n)= \begin{cases}\left(\begin{array}{c}
n+2 \\
2
\end{array}\right), & n=0, \ldots, t-1, \\
\left(\begin{array}{c}
t+2 \\
2
\end{array}\right), & n \geq t .\end{cases}
$$

We denote by $\mathbf{n}$ the maximal ideal of $\mathcal{O}_{X}$. Since $X$ has maximal Hilbert function, we have that the pair $\mathbf{n},(\bar{x})$ satisfies $\mathcal{V}_{n}^{2}$ for $n=0, \ldots, t, \delta=0$, and $G(\mathbf{n})$ is CohenMacaulay. From this it is easy to see that $R=\mathcal{O}_{X}\left[T_{1}, \ldots, T_{d-1}\right]_{\left(T_{1}, \ldots, T_{d-1}\right)}$, where $\mathbf{m}$ is a maximal ideal of $R$, and $J=\left(\bar{x}, T_{1}, \ldots, T_{d-1}\right)$ satisfy the conditions of the claim.

Case $\delta=1, \varepsilon=d-1$. Let us consider the closed subscheme $X$ of $\left(\mathbf{k}^{3}, 0\right)$ defined by the maximal minors of

$$
\left(\begin{array}{cc}
0 & z \\
z & y^{e-2} \\
y & x^{\alpha}
\end{array}\right)
$$

with $\alpha \leq e-2$. From the Hilbert-Burch theorem we have that $I(X)$ is a height two perfect ideal of $S$, i.e. $X$ is a curve singularity. An easy computation gives us

$$
H_{X}(n)= \begin{cases}n+2, & n=1, \ldots, \alpha, \\ n+1, & n=\alpha+1, \ldots, e-1, \\ e, & n \geq e-1 .\end{cases}
$$

From now on we set $e=t+2$ and $\alpha=t-1$. Let $Y$ be the curve singularity obtained by union of $X$ and $\left(\begin{array}{c}t+1 \\ 2\end{array}\right)-(t+1)$ generic straight lines through the origin. 
From [Eli93a], Proposition 2.2, we get

$$
H_{Y}(n)= \begin{cases}\left(\begin{array}{c}
n+2 \\
2
\end{array}\right), & n=0, \ldots, t-1, \\
\left(\begin{array}{c}
t+1 \\
2
\end{array}\right), & n=t, \\
\left(\begin{array}{c}
t+1 \\
2
\end{array}\right)+1, & n \geq t+1 .\end{cases}
$$

Notice that $I(Y) \subset(x, y, z)^{t}$. Hence if $\mathbf{n}$ is the maximal ideal of $\mathcal{O}_{Y}$, then the pair $\mathbf{n}, \bar{x}$ satisfies $\mathcal{V}_{n}^{2}$ for $n=0, \ldots, t$, and

$$
\begin{aligned}
\operatorname{dim}_{\mathbf{k}}\left(\mathbf{n}^{t+1} / \bar{x} \mathbf{n}^{t}\right) & =\operatorname{dim}_{\mathbf{k}}\left(\mathbf{n}^{t+1} / \bar{x} \mathbf{n}^{t+1}\right)-\operatorname{dim}_{\mathbf{k}}\left(x \mathbf{n}^{t} / \bar{x} \mathbf{n}^{t+1}\right) \\
& =e_{0}(X)-\operatorname{dim}_{\mathbf{k}}\left(\mathbf{n}^{t} / \mathbf{n}^{t+1}\right) \\
& =1
\end{aligned}
$$

[Lip71], Remark (a) and (b) to Corollary 1.10. Notice that $H_{Y}(t-1)=H_{Y}(t)$, so $G(\mathbf{n})$ is not Cohen-Macaulay. The local ring $R=\mathcal{O}_{Y}\left[T_{1}, \ldots, T_{d-1}\right]_{\left(T_{1}, \ldots, T_{d-1}\right)}$, where $\mathbf{m}$ is a maximal ideal of $R$, and $J=(\bar{x})$ satisfy the conditions of the claim.

Case $\delta=1, \varepsilon=d$. Let $X$ be a curve singularity of $\left(\mathbf{k}^{3}, 0\right)$ formed by the union of $\left(\begin{array}{c}t+1 \\ 2\end{array}\right)$ generic straight lines throughout the origin. The Hilbert function of $X$ is maximal:

$$
H_{X}(n)= \begin{cases}\left(\begin{array}{c}
n+2 \\
2
\end{array}\right), & n=0, \ldots, t-1, \\
\left(\begin{array}{c}
t+1 \\
2
\end{array}\right), & n \geq t .\end{cases}
$$

In particular, $G(\mathbf{n})$ is Cohen-Macaulay. The $\operatorname{ring} R=\mathcal{O}_{X}\left[T_{1}, \ldots, T_{d-1}\right]_{\left(T_{1}, \ldots, T_{d-1}\right)}$ satisfies the conditons of the claim.

Example 2.10. It is worthwhile to recall an example given by Guerrieri, [Gue94]: let $R=\mathbf{k}[x, y, z, u]_{(x, y, z, u)}, \mathbf{k}$ an infinite field. Let $I=\left(x^{2}, y^{2}, z^{2}, u^{2}, x y+z u\right)$ and $J=\left(x^{2}, y^{2}, z^{2}, u^{2}\right)$. Then the pair $I, J$ satisfies $\mathcal{V}_{n}^{2}$ for all $n$ but $n=3$. This example shows us that condition $\mathcal{V}_{n}^{2}$ does not imply $\mathcal{V}_{n+1}^{2}$; in fact, the examples with $\delta=1$ in the last propostion do not satisfy $\mathcal{V}_{t+1}^{2}$.

\section{The growth of the Hilbert function}

This section is devoted to study the growth of the Hilbert function of an $\mathbf{m}-$ primary ideal under the conditions of Theorem 2.7, and we will apply it to the study of Hilbert functions in the one-dimensional case.

Given a numerical function $F: \mathbb{N} \longrightarrow \mathbb{N}$, we will denote by $\Delta^{r} F$ its $r$-th derivative, $r \geq 1$, i.e. $\Delta F(n)=F(n)-F(n-1)$ and by induction $\Delta^{r+1} F=\Delta\left(\Delta^{r} F\right)$. We put $\Delta^{0} F=F$.

Lemma 3.1. Let $R$ be a one-dimensional Cohen-Macaulay local ring. Let $I$ be an $\mathbf{m}$-primary ideal of $R$, and let $x$ be a superficial element of $I$. We denote by $\mathcal{H}$ the numerical function defined by $\mathcal{H}(n)=$ length $_{A}\left(I^{n} / x I^{n-1}\right)$. Then

$$
H_{I}(n)-H_{I}(n-1)=\mathcal{H}(n)-\mathcal{H}(n+1) .
$$

Proof. Let us consider the following exact sequence of $R$-modules:

$$
0 \longrightarrow \frac{x I^{n-1}}{x I^{n}} \longrightarrow \frac{I^{n}}{x I^{n}} \longrightarrow \frac{I^{n}}{x I^{n-1}} \longrightarrow 0 .
$$


We have

$$
\begin{aligned}
\operatorname{length}_{R}\left(\frac{I^{n}}{x I^{n-1}}\right) & =\text { length }_{R}\left(\frac{I^{n}}{x I^{n}}\right)-\text { length }_{R}\left(\frac{x I^{n-1}}{x I^{n}}\right) \\
& =e_{0}(I)-\text { length }_{R}\left(\frac{I^{n-1}}{I^{n}}\right) \\
& =e_{0}(I)-H_{I}(n-1)
\end{aligned}
$$

([Lip71], Remark (a) and (b) to Corollary 1.10). Hence we have

$$
H_{I}(n)-H_{I}(n-1)=\mathcal{H}(n)-\mathcal{H}(n+1),
$$

for all $n \geq 0$.

Proposition 3.2. Let $R$ be a d-dimensional, $d \geq 1$, Cohen-Macaulay local ring. Let $I$ be an $\mathbf{m}$-primary ideal of $R$, and $J=\left(x_{1}, \ldots, x_{d}\right)$ a minimal reduction of $I$, with $x_{1}, \ldots, x_{d}$ a superficial sequence of $I$. Let us assume that there exists an integer $t \geq 1$ such that

- I, $J$ satisfy the condition $\mathcal{V}_{n}^{2}$ for $n=0, \ldots, t$, and

- length $\left(I^{t+1} / J I^{t}\right) \leq 2$.

Then $H_{I /\left(x_{1}, \ldots, x_{d-1}\right)}$ is non-decreasing.

Proof. Since the conditions of the claim are preserved modulo superficial sequences, we may assume $d=1$. We set $J=(x)$.

Let us consider the morphisms

$$
\frac{I^{n-2}}{I^{n-1}} \stackrel{\cdot x}{\longrightarrow} \frac{I^{n-1}}{I^{n}} .
$$

Since the pair $I, J$ satisfies $\mathcal{V}_{n}^{2}$ for $n=0, \ldots, t$, we get that these morphisms are mono, so

$$
H_{I}(n-1) \leq H_{I}(n)
$$

for $n \leq t-1$.

We need to consider two cases:

Case 1: $\mathcal{H}(t) \leq 1$. Then by Proposition 2.3 we get

$$
1 \geq \mathcal{H}(n) \geq \mathcal{H}(n+1) \geq 0
$$

for all $n \geq t$. By Lemma 3.1 we obtain the claim.

Case 2: $\mathcal{H}(t) \geq 2$. We set $\omega=\operatorname{length}_{R}\left(I^{t+1} / x I^{t}+I^{t+2}\right)$, and consider the projection

$$
\frac{I^{t+1}}{x I^{t}} \longrightarrow \frac{I^{t+1}}{x I^{t}+I^{t+2}}
$$

If $\omega=0$ then $I^{t+1}=x I^{t}$, and $H_{I}(n)=e_{0}(I)$ for all $n \geq t$, [Lip71], Theorem 1.9. Hence the Hilbert function is non-decreasing.

If $\omega=2$, then the projection is an isomorphism and $I^{t+2} \subset x I^{t}$. Hence we have that for all $n \geq t$ the natural projection induces isomorphisms

$$
\frac{I^{n+1}}{x I^{n}} \longrightarrow \frac{I^{n+1}}{x I^{n}+I^{n+2}} .
$$


Since $G(I) / x^{*} T G(I)$ is a standard $R / I$-algebra, and $\omega \leq 2$, we get that $\mathcal{H}(n)$ is non-increassing for $n \geq t$, [Bla95], [BN96]. From Lemma 3.1 we get that $H_{I}$ is non-decreasing.

Let us assume $\omega=1$. From Proposition 2.3 there exists $g \in I$ such that the coset of $g^{n+1}$ in $I^{n+1} / x I^{n}+I^{n+2}$ is a generator for $n \geq t$. Hence the coset of $g^{n+1}$ in $I^{n+1} / x I^{n}$ is also a generator, and then the morphism

$$
\frac{I^{n+1}}{x I^{n}} \stackrel{. g}{\longrightarrow} \frac{I^{n+2}}{x I^{n+1}}
$$

is an epimorphism for $n \geq t$. From Lemma 3.1 we get that $H_{I}$ is non-decreasing.

We will denote by $P_{I} \in \mathbb{Z}[Z]$ the Poincaré series of $I$ :

$$
P_{I}(Z)=\sum_{n \geq 0} H_{I}(n) Z^{n}
$$

It is well known that there exists a polynomial $f(Z) \in \mathbb{Z}[Z]$ such that

$$
P_{I}(Z)=\frac{f(Z)}{(1-Z)^{d}}
$$

Proposition 3.3. Let $R$ be a d-dimensional, $d \geq 1$, Cohen-Macaulay local ring. Let $I$ be an $\mathbf{m}$-primary ideal of $R$, and $J=\left(x_{1}, \ldots, x_{d}\right)$ a minimal reduction of $I$. Let us assume that there exists an integer $t \geq 1$ such that

- $I, J$ satisfy the condition $\mathcal{V}_{n}^{2}$ for $n=0, \ldots, t$, and

- $\delta=\operatorname{length}\left(I^{t+1} / J I^{t}\right) \leq 1$.

We consider the integer $\bar{\delta}=$ length $\left(I^{t+1}+\left(x_{2}, \ldots, x_{d}\right)\right) /\left(J I^{t}+\left(x_{2}, \ldots, x_{d}\right)\right) \leq 1$. Then

(1) If $\bar{\delta}=0$, then $G(I)$ is Cohen-Macaulay and there exists $u \leq t$ such that

$$
P_{I}=\frac{a_{0}+a_{1} Z+\cdots+a_{u} Z^{u}}{(1-Z)^{d}}
$$

with $a_{i}>0$ for all $i \leq u$.

(2) If $\bar{\delta}=1$, then

$$
P_{I}=\frac{a_{0}+a_{1} Z+\cdots+a_{t} Z^{t}+Z^{u}}{(1-Z)^{d}}
$$

with $u>t, a_{i}>0$ for all $i \leq t-1$, and $a_{t} \geq 0$. If $G(I)$ is Cohen-Macaulay then $a_{t}>0$, and $u=t+1$.

In particular, $\Delta^{d-1} H_{I}$ is non-decreasing.

Proof. From [Sin74] and Theorem 2.7 we have

$$
\Delta^{d-1} P_{I}=P_{\bar{I}}
$$

with $\bar{R}=R /\left(x_{2}, \ldots, x_{d}\right), \bar{I}=I /\left(x_{2}, \ldots, x_{d}\right), \bar{J}=J /\left(x_{2}, \ldots, x_{d}\right)$, and $P_{\bar{I}}=$ $\left(a_{0}+a_{1} Z+\cdots+a_{s} Z^{s}\right) /(1-Z)$. Notice that $a_{n}=H_{\bar{I}}(n)-H_{\bar{I}}(n-1)$ for all $n \geq 0$.

If $\bar{\delta}=0$ then the pair $\bar{I}, \bar{J}$ satisfies $\mathcal{V}_{n}^{2}$ for all $n \geq 0$. From the Vallabrega-Valla result we get that $G(\bar{I})$ is Cohen-Macaulay, and $G(I)$ as well. From this it is easy to prove (1). 
Suppose $\bar{\delta}=1$. From Lemma 3.1 and Proposition 3.2 we have that for all $n \geq 0$

$$
a_{n}=H_{\bar{I}}(n)-H_{\bar{I}}(n-1)=\mathcal{H}_{\bar{I}}(n)-\mathcal{H}_{\bar{I}}(n+1) .
$$

Since the pair $\bar{I}, \bar{J}$ satisfies $\mathcal{V}_{n}^{2}$ for $n=0, \ldots, t$ and $\bar{\delta} \neq 0$, from (F2) we get that the morphism, $n=0, \ldots, t-1$,

$$
\frac{\bar{I}^{n-1}}{\bar{I}^{n}} \stackrel{x_{1}}{\longrightarrow} \frac{\bar{I}^{n}}{\bar{I}^{n+1}}
$$

is mono, but not an isomorphism. Hence $a_{n}>0$ for all $n=0, \ldots, t-1$.

On the other hand we have that $\mathcal{H}_{\bar{I}}(n) \leq 1$ for all $n \geq t+1$, by Proposition 2.3. From this and (F2) we get the first part of (2). The second part of (2) is clear.

One-dimensional case. Let $R$ be a one-dimensional equicharacteristic CohenMacaulay local ring. We will denote by $b=\operatorname{dim}_{\mathbf{k}}\left(\mathbf{m} / \mathbf{m}^{2}\right)$ the embedding dimension of $R$. Recall that Abhyankar proved that $e_{0}(R) \geq b$.

It is a longstanding problem to find conditions to assure that the Hilbert function of $R$ is non-decreasing. It is well known that for $b \leq 2$ the Hilbert function $H_{R}$ is non-decreasing. The main open problem was the conjecture of Sally: if $b=3$ then the Hilbert function of $R$ is non-decreasing, [Sal78]. This conjecture was settled in [Eli93a]. See [GR83] for examples of one-dimensional local rings with decreasing Hilbert functions with $b \geq 4$, and [Eli93a], [Eli94c] for further results. As a corollary of Proposition 3.2 we obtain that for $e_{0}(R) \leq b+2$ the Hilbert function of $R$ is non-decreasing.

We can summarize the above results:

Proposition 3.4. Let $R$ be a equicharacteristic one-dimensional Cohen-Macaulay local ring of multiplicity $e_{0}$ and embedding dimension $b$. Then $1 \leq b \leq e_{0}$, and the Hilbert function of $R$ is non-decreasing in the following cases:

(1) $b \leq 3$, or

(2) $b \leq e_{0}(R) \leq b+2$.

The first open case for which we don't know if the Hilbert function is decreasing is $b=4$ and $e_{0}=7$. Recall that the first known example with a decreasing Hilbert function for $b=4$ has multiplicity $e_{0}(R)=32$, [GR83].

It is well known that if $b=2$ or $e$, then the tangent cone is Cohen-Macaulay, [Sal77]. The first case for which the associated graded ring can be non-CohenMacaulay is $b=3, e=4$. In this case $e_{1}=4$ or 5 , [Eli90], and the Hilbert function is determined by this coefficient, [Eli96]:

- $e_{1}=4, H=\{1,3,4, \ldots\}, G(R)$ Cohen-Macaulay, example: $R=\mathbf{k}\left[\left[t^{4}, t^{5}, t^{6}\right]\right]$,

- $e_{1}=5, H=\{1,3,3,4, \ldots\}, G(R)$ not Cohen-Macaulay, example: $R=\mathbf{k}\left[\left[t^{4}, t^{5}, t^{11}\right]\right]$.

\section{REFERENCES}

[Abh67] Abhyankar, S.S. Local rings of high embedding dimension. Amer. J. Math., 89:10731077, 1967. MR 36:3775

[Bla95] Blancafort, C. Hilbert functions of graded algebras over Artinian rings. Pure and Applied Alg., 125:55-78, 1998.

[BN96] Blancafort, C. and Nollet, S. Hilbert polynomials over Artinian local rings. Preprint, 1996. MR 98m: 13023 
[Eli90] Elias, J. Characterization of the Hilbert-Samuel polynomials of curve singularities. Compositio Math., 74:135-155, 1990. MR 91h:13016

[Eli93a] Elias, J. The conjecture of Sally on the Hilbert function for curve singularities. J. of Algebra, 160(1):42-49, 1993. MR 94j:13018

[Eli94c] Elias, J. Roller Coaster Curve Singularities. J. of Algebra, 168(3):864-867, 1994. MR 95e: 13013

[Eli96] Elias, Juan. The regularity index and the depth of the tangent cone of curve singularities. Japan J. Math., 22(1):51-68, 1996. MR 97f:13004

[ES76] Eakin, P. and Sathaye, A. Prestable ideals. J. of Algebra, 41:439-454, 1976. MR 54:7449

[Gue94] Guerrieri, A. On the depth of the associated graded ring of an m-primary ideal of a Cohen-Macaulay local ring. J. of Algebra, 167:745-757, 1994. MR 95h:13004

[Gue95] Guerrieri, A. On the depth of the associated graded ring. Proc. A.M.S., 123:11-20, 1995. MR 95c: 13002

[GR83] Gupta, S.K. and Roberts, L.G. Cartesian squares and ordinary singularities of curves. Comm. in Algebra, 11(2):127-182, 1983. MR 84h:13036

[HLS92] Heinzer, W., Lantz, D., and Shah, K. The Ratliff-Rush ideals in a Noetherian ring. Comm. in Algebra, 20(2):591-622, 1992. MR 93c: 13002

[HM94] Huckaba, S. and Marley, T. Hilbert coefficients and the depths of associated graded rings. J. London Math. Soc., 56:64-76, 1997. MR 98i:13028

[Huc96] Huckaba, S. A d-dimensional extension of a lemma of Huneke's and formulas for the Hilbert coefficients. Proc. A.M.S., 124:1393-1401, 1996. MR 96g:13018

[Huc97] Huckaba, S. On associated graded rings having almost maximal depth. Comm. Algebra 26:967-976 (1998). CMP 98:08

[Ito95] Itoh, S. Hilbert coefficients on integrally closed ideals. J. of Algebra, 176:638-652, 1995. MR 96g: 13019

[Lip71] Lipman, J. Stable ideals and Arf rings. Amer. J. of Math., 93:649-685, 1971. MR 44:203

[RosV96a] Rossi, M.E. and Valla, G. On a conjecture of Sally. Comm. in Algebra, 24:4249-4261, 1996. MR 97j:13021

[SV74] Sally, J. and Vasconcelos, W.V. Stable rings. J. Pure and Appl. Alg., 4:319-336, 1974. MR 53:13185

[Sal77] Sally, J. On the associated graded ring of a local Cohen-Macaulay ring. J. Math. Kyoto Univ., 17:19-21, 1977. MR 56:8555

[Sal78] Sally, J. Number of generators of ideals in local rings. Lec. Notes in Pure and Appl. Math., 35, Marcel Dekker, New York, 1978. MR 58:5654

[Sal79a] Sally, J. Cohen-Macaulay local rings of maximal embedding dimension. J. of Algebra, 56:168-183, 1979. MR 80e:14022

[Sal79b] Sally, J. Super-regular sequences. Pacific J. Math., 84:465-481, 1979. MR 81m:13024

[Sal79c] Sally, J.D. Stretched Gorenstein rings. J. London Math. Soc., 20(2):19-26, 1979. MR 80k:14006

[Sal80b] Sally, J. Good embedding dimensions for Gorenstein singularities. Math. Ann., 249:95106, 1980. MR 82c: 13031

[Sal80a] Sally, J. Tangent cones at Gorenstein singularities. Compositio Mathematica, 40(2):167-175, 1980. MR 81e:14004

[Sal83] Sally, J. Cohen-Macaulay local rings of embedding dimension $e+d-2$. J. of Algebra, 83:393-408, 1983. MR 85c: 13017

[Sal92] Sally, J. Hilbert coefficients and reduction number 2. J. Algebraic Geometry, (1):325333, 1992. MR 93b:13026

[Sin74] Singh, B. Effect of a permisible blowing-up on the local Hilbert function. Inv. Math., 26:201-212, 1974. MR 50:4584

[VV78] Vallabrega, P. and Valla, G. Form rings and regular sequences. Nagoya Math. J., 72:93-101, 1978. MR 80d:14010

[Val79] Valla, G. On form rings which are Cohen-Macaulay. J. of Algebra, pages 247-250, 1979. MR 80h: 13025

[Vas94] Vasconcelos, W.V. Hilbert functions, analytic spread, and Koszul homology. Contemp. Math., 159:401-422, 1994. MR 95a:13006 
[Vaz95] Vaz Pinto, M.T.R. Structure of Sally modules and Hilbert functions. PhD thesis, Rutgers University, 1995.

[Wan97] Wang, H. On Cohen-Macaulay local rings with embedding dimension $e+d-2$. J. of Algebra, 190:226-240, 1997. MR 98d:13027

Departament d’Àlgebra i Geometria, Universitat de Barcelona, Gran Via 585, 08007 Barcelona, Spain

E-mail address: elias@cerber.mat.ub.es 